Ana Katherine da Silveira Gonçalves ${ }^{1}$

ana Carla Gomes Canário²

Patrícia Uchôa leitão Cabral ${ }^{2}$

Rayanna Assunção Henrique da Silva ${ }^{3}$

Maria Helena Constantino Spyrides ${ }^{4}$

Paulo César Giraldo 5

JOSÉ ELEUTÉRIO JR. ${ }^{6}$

Artigo original

Palavras-chave

Exercício

Qualidade de vida

Mulheres

Envelhecimento

Menopausa

Saúde da mulher

Keywords

Exercise

Quality of life

Women

Aging

Menopause

Women's health

Programa de Pós-graduaç̃o em Ciências da Saúde da Universidade Federal do Rio Grande do Norte - UFRN

Ana Katherine da Silveira Goncalves Avenida Gal. Gustavo Cordeiro de Farias s/n

CEP: 59010-180

Natal (RN), Brasil

Recebido

20/10/2011

Aceito com modificacōes

$17 / 11 / 2011$

\section{Impacto da atividade física na qualidade de vida de mulheres de meia idade: estudo de base populacional}

\author{
Impact of physical activity on quality of life in middle-aged women: \\ a population based study
}

\section{Resumo}

OBJETIVO: Avaliar o impacto da prática de atividade física na qualidade de vida de mulheres de meia idade. MÉTODOS: Estudo de base populacional e corte transversal, que incluiu uma amostra estratificada de 370 mulheres de meia idade entre 40 a 65 anos, recrutadas a partir de uma população de 20.801 mulheres atendidas no período de um ano nas redes básicas de saúde, inseridas nos quatro distritos (Norte, Sul, Leste e Oeste) que compõem o sistema de saúde da cidade de Natal, Rio Grande do Norte, de junho a setembro de 2011 . O cálculo da amostra teve por base um nível de confiança de $95 \%$, com poder do teste de $80 \%$, erro de estimativa de $5 \%$ e considerou-se a proporção de pacientes classificadas com qualidade de vida adequada (indicador $\geq 26$ ) da amostra piloto. Os dados foram coletados enquanto as mulheres aguardavam na sala de espera para a consulta de rotina. Para avaliar a qualidade de vida geral, utilizou-se a versão abreviada do WHOQOL (WHOQOL-Bref-WHO Quality of Life - BREF), e sua relação com os sintomas do climatério foi avaliada por meio do Menopause Rating Scale (MRS). O nível de atividade física foi avaliado pelo questionário International Physical Activity Questionnaire (IPAQ), versão curta, semana usual. Para obter-se a classificação dos níveis de atividade física, utilizaram-se três categorias: sedentária, moderadamente ativa e muito ativa. A análise estatística foi realizada utilizando o programa estatístico Minitab, versão 16. RESULTADOS: A média de idade das mulheres foi de 49,8 anos $( \pm 8.1)$, foram predominantemente caucasianas $(72,7 \%)$, casadas $(61,6 \%)$, não fumantes $(93,5 \%)$ e com o Ensino Médio completo $(47,8 \%)$. Considerando os domínios presentes no WHOQOL-Bref para avaliar qualidade de vida, os escores foram significativamente diferentes entre os grupos de mulheres sedentárias, moderadamente ativas e muito ativas $(p<0,01)$. Em relação à atividade física e aos sintomas do climatério, foram observadas diferenças significativas para todos os domínios: psicológico $(p<0,01)$, somáticovegetativo $(p<0,01)$ e urogenital $(p<0,01)$. CONCLUSÃO: A prática de atividade física melhora significativamente a qualidade de vida das mulheres de meia idade.

\section{Abstract}

PURPOSE: To evaluate the influence of physical activity on the quality of life of middle-aged women. METHODS: A population-based cross-sectional study was conducted on 370 women aged 40 to 65 years-old recruited from a population-based sample. Enrollment took place in Basic Health Units in each health district of the city (North, South, East, and West) from June to September 2011 . According to the Municipal Health Department of the City, 20,801 women were assisted at the Basic Health Units during a one-year period. The sample size calculation was stratified by district and based on a $95 \%$ confidence level with a power of $80 \%$, as well as an error estimate of $5 \%$ and it was considered proportional to the number of patients classified as having adequate quality of life (indicator $\geq 26$ ) in the general population. Data were collected while women waited for their routine appointment at the Health Unit. WHOQOLBref was used to evaluate the quality of life, and menopause rating scale (MRS) was used to determine climacteric symptoms. The level of physical activity was assessed by means of the International Physical Activity Questionnaire (IPAQ). To obtain the classification of PA levels, we used three categories: sedentary, moderately active, and very active. Statistical analysis was performed using the Minitab software, version 16. RESULTS: The mean age of the subjects was 49.8 years-old $( \pm 8.1)$ and they were predominantly Caucasian $(72.7 \%)$, married $(61.6 \%)$, non-smokers $(93.5 \%)$,

Programa de Pós-graduação em Ciências da Saúde da Universidade Federal do Rio Grande do Norte - UFRN - Natal (RN), Brasil. Professora do Programa de Pós-graduação em Ciências da Saúde da Universidade Federal do Rio Grande do Norte - UFRN - Natal (RN), Brasil.

${ }^{2}$ Aluna do Programa de Pós-graduação em Ciências da Saúde da Universidade Federal do Rio Grande do Norte - UFRN - Natal (RN), Brasil.

3 Aluna de graduação do Curso de Estatística da Universidade Federal do Rio Grande do Norte - UFRN - Natal (RN), Brasil. ${ }_{4}^{4}$ Professora Adjunta do Departamento de Estatística da Universidade Federal do Rio Grande do Norte - UFRN - Natal (RN), Brasil. 5 Professor Titular do Departamento de Tocoginecologia da Universidade Estadual de Campinas - UNICAMP - Campinas (SP), Brasil. - Professor Adjunto do Departamento Materno-infantil da Faculdade de Medicina da Universidade Federal do Ceará - UFC - Fortaleza (CE), Brasil.

Conflito de interesses: não há 
and had High School education (47.8\%). Using the WHOQOL, mean scores were found to be significantly different between the groups (low, moderate, and vigorous physical activity), classified according to the domains of quality of life $(p<0.01)$. Concerning physical activity and climacteric symptoms, significant differences were found for all domains: psychological $(p<0.01)$, vegetative-somatic ( $<<0.01)$, and urogenital (p<0.01). CONCLUSION: Physical activity improves quality of life in middle-aged women.

\section{Introdução}

O envelhecimento da população mundial representa uma das mais notáveis histórias de sucesso da Medicina e da humanidade, mas é também uma fonte de vários desafios. Diversas intervenções físicas e cognitivas têm sido realizadas, com o intuito de minimizar os efeitos deste evento ${ }^{1,2}$.

Este processo não é influenciado apenas pelo tempo. O estilo de vida individual de cada pessoa parece ser determinante juntamente com aspectos psicológicos, sociais, biológicos e funcionais, que estão inseridos neste contexto ${ }^{1-6}$. Em termos biológicos, são observadas, principalmente, alterações no sistema cardiorrespiratório e neuromuscular. Estas modificações, somadas a outras decorrentes do envelhecer, podem acelerar a ocorrência de doenças como osteoporose e aterosclerose ${ }^{3}$, contribuir para desencadear hiperlipidemia ${ }^{4}$ e doença cardiovascular ${ }^{5}$. Ocorre, ainda, um declínio na capacidade física e mental que frequentemente compromete as atividades diárias ${ }^{6,7}$. Nas mulheres, além das alterações fisiológicas advindas da idade e inatividade física, ocorrem outras em função da menopausa. O desequilíbrio hormonal inerente a esta fase promove uma plêiade de sintomas físicos e psicológicos inerentes à menopausa ${ }^{2-8}$.

A despeito de tratar-se de um processo fisiológico que prepara a mulher para senectude, as alterações hormonais advindas deste processo efetivamente comprometem a qualidade de vida $(\mathrm{QV})$ das mulheres ${ }^{6-8}$. Esses distúrbios se manifestam principalmente na forma de sintomas vasomotores (calores e suor noturno) e psicológicos, tais como: irritabilidade, insônia, diminuição da libido e oscilações de humor. Apesar destas alterações se limitarem a alguns grupos mais vulneráveis de mulheres, de um modo geral, os distúrbios psíquicos e emocionais podem causar impacto negativo na QV das mulheres ${ }^{6,8,9}$. Por outro lado, estudos demonstram que a prática de atividade física pode causar melhora de muitos destes sintomas, em particular, a insônia, o humor e as dores musculares ${ }^{6-10}$.

Importantes estudos têm demonstrado os inúmeros benefícios da prática de atividade física ${ }^{8-11}$. Mulheres menopausadas, que seguiram as recomendações preconizadas pelo Colégio Americano de Medicina dos Esportes e da Associação Americana do Coração, nos quais incluía a prática de atividade física moderada pelo tempo mínimo de 30 minutos, cinco vezes por semana, ou atividade física intensa pelo tempo mínimo de 20 minutos, três vezes por semana, apresentaram níveis significativos de melhora na
$\mathrm{QV}^{8}$. Resultados semelhantes também foram encontrados em um estudo recente com mulheres brasileiras de mais de 60 anos $^{11}$.

Em um estudo de revisão sistemática que selecionou 14 artigos, a prática de atividade física esteve consistentemente associada à melhora da $\mathrm{QV}$. A prática de atividade física também esteve fortemente associada ao bem-estar psicológico ${ }^{7}$. Estudo de metanálise com 49 ensaios clínicos controlados observou decréscimo de $48 \%$ nos níveis de ansiedade no grupo de estudo, quando comparado ao Grupo Controle9. Um ensaio clínico randomizado avaliou especificamente sintomas psicológicos e concluiu que a prática de atividade física pode minimizar sintomas de depressão e estresse, melhorando consequentemente $\mathrm{a} \mathrm{QV}^{12}$.

Apesar de serem reconhecidos os inúmeros benefícios da prática de atividade física na QV de homens e mulheres, esta ainda ocorre apenas de forma isolada e pontual para algumas mulheres, existindo, portanto, a necessidade da realização de estudos que divulguem a importância deste tema. O presente estudo propõe avaliar o impacto da prática de atividade física na $\mathrm{QV}$ de mulheres de meia idade.

\section{Métodos}

Trata-se de um estudo de corte transversal de base populacional. A população-alvo foi constituída por mulheres consideradas de meia idade ${ }^{13}$, com idades entre 40 a 65 anos, residentes na cidade de Natal, no Rio Grande do Norte, Brasil, assistidas nas Unidades Básicas de Saúde de cada distrito sanitário da Cidade (Norte, Sul, Leste e Oeste), de junho a setembro de 2011.

A população atendida nos setores de ginecologia das Unidades Básicas de Saúde em todos os distritos da Cidade consistiu em 20.801 mulheres, de acordo com a Secretaria Municipal de Saúde da Cidade. O tamanho amostral foi estabelecido em 365 mulheres, porém foram respondidos e concluídos 370 questionários válidos, prevendo-se possíveis perdas. O cálculo da amostra teve por base um nível de confiança de $95 \%$, com poder do teste de $80 \%$, erro de estimativa de $5 \%$ e considerou-se a proporção de pacientes classificadas com QV adequada (indicador $\geq 26$ ) da amostra piloto.

Estabeleceram-se como critérios de inclusão: mulheres consideradas pela Organização Mundial de Saúde (OMS) como de meia idade ( 40 a 65 anos ${ }^{13}$ aparentemente saudáveis, que não estivessem fazendo uso de terapia de reposição hormonal. 
As mulheres que apresentaram alterações neurológicas, doenças psiquiátricas, endócrinas e usuárias de medicamentos que apresentam interferência na QV (por exemplo: tratamento hormonal, antidepressivos, ansiolíticos, neurolépticos) foram excluídas do presente estudo.

Posteriormente à determinação dos critérios de inclusão e exclusão, as mulheres elegíveis foram convidadas a participar do estudo. Após os esclarecimentos, as mulheres que desejaram participar do estudo assinaram o termo de consentimento livre e esclarecido (TCLE). Enquanto aguardava sua consulta na Unidade de Saúde, cada participante recebeu um questionário para registro de dados pessoais, como: idade, cor da pele, tempo de menopausa, terapia de reposição hormonal, anos de escolaridade, estado civil, renda e questionários validados autoaplicáveis, referentes à QV e ao nível de atividade física. Utilizou-se a classificação do Censo Demográfico do Instituto Brasileiro de Geografia e Estatística (IBGE) para a determinação da faixa de renda, que é dada na quantidade de salários mínimos ${ }^{14}$.

Adotou-se como definição de atividade física aquele determinado pela OMS, cuja atividade física seria todo movimento corporal produzido pela musculatura esquelética que resulta na dispersão de energia, incluindo exercícios de condicionamento, atividades ocupacionais e atividade de casa ${ }^{15}$.

O nível de atividade física foi avaliado pelo questionário International Physical Activity Questionnaire (IPAQ), versão curta, semana usual, validada no Brasil ${ }^{16}$. O IPAQ avalia a frequência, em dias, e a duração, em minutos, das atividades realizadas como lazer, ocupacionais, locomoção e trabalho doméstico por mais de dez minutos contínuos durante uma semana normal ${ }^{16}$. A pontuação foi obtida pela soma da quantidade de dias e minutos ou horas das atividades realizadas na semana anterior ao preenchimento do questionário. Para obter-se a classificação dos níveis de atividade física, utilizaram-se três categorias: sedentária, moderadamente ativa e muito ativa.

Para avaliar a QV geral, utilizou-se a versão abreviada em português do Instrumento de Avaliação de QV da OMS, o WHOQOL-Bref ${ }^{17}$. Esse instrumento contém 26 questões distribuídas em quatro domínios: relações sociais, psicológico, físico e meio ambiente. Cada domínio é composto por questões cujas pontuações das respostas variam entre um e cinco. A versão em português do instrumento apresentou características satisfatórias de consistência interna, validade discriminante, validade de critério, validade concorrente e fidedignidade testereteste ${ }^{17}$. A QV e os sintomas do climatério foram avaliados mediante a utilização do Menopause Rating Scale (MRS), que quantifica a gravidade dos sintomas nos domínios psicológico, somático-vegetativo e urogenital ${ }^{18 .}$

Inicialmente, desenvolveu-se a análise exploratória dos dados, descrevendo as médias e os desvios padrão das variáveis quantitativas envolvidas no estudo, bem como de frequências relativas das variáveis categorizadas. Em seguida, procedeu-se ao teste do $\chi^{2}$ de Pearson com o intuito de verificar possíveis associações entre as variáveis sociodemográficas e o nível de atividade física das mulheres.

Com o objetivo de classificar as mulheres em período menopausal quanto à $\mathrm{QV}$, utilizou-se uma técnica multivariada, denominada análise por cluster, que classifica os sujeitos de acordo com o comportamento de variáveis simultâneas, neste caso consideraram-se os domínios do WHOQOL-Bref ${ }^{17}$. Nesta análise, considerou-se o método de ligação de Ward e a distância Euclidiana para caracterizar os grupos quanto às similaridades dos indivíduos, resultando na classificação das mulheres em três categorias: baixa, média e alta QV, de acordo com a similaridade.

Para captar a associação entre variáveis categorizadas ordinais (atividade física e QV), utilizou-se o teste de Mantel-Haenszel. Procedeu-se à análise de variância (ANOVA) e aos testes de Tukey para comparação de médias entre as categorias de QV. Considerou-se o nível de significância de $5 \%$ para os testes. O programa estatístico utilizado foi o Minitab, versão $16^{19}$.

Este estudo foi previamente aprovado pelo Comitê de Ética em Pesquisa da Universidade Federal do Rio Grande do Norte (UFRN) - 130/11 CEP-UFRN.

\section{Resultados}

A média de idade das mulheres que participaram deste estudo foi de 49,8 ( $\pm 8,1$ anos), sendo que $28,4 \%$ apresentavam idade igual ou superior a 55 anos. Predominantemente brancas $(72,7 \%)$, casadas $(61,6 \%)$, não fumantes $(93,5 \%)$, com Ensino Médio completo $(47,8 \%)$ e renda de dois salários mínimos $(70,8 \%)$, como pode ser visto na Tabela 1. Quanto à classificação dos níveis de atividade física, observou-se que $132(35,7 \%)$ eram ativas, $129(34,8 \%)$ eram moderadamente ativas e $109(29,4 \%)$ eram sedentárias.

A Tabela 1 mostra a distribuição das mulheres segundo os níveis de atividade física e características sociodemográficas. Detectou-se diferença significativa no comportamento $\mathrm{da}$ atividade física quanto à renda $(\mathrm{p}=0,01)$, indicando que as mulheres com renda mensal entre dois e quatro salários mínimos apresentaram um percentual maior de ativas $(45,4 \%)$. Para as demais características não houve diferença significativa.

$\mathrm{Na}$ Tabela 2 constatou-se que as mulheres que praticam mais atividades físicas têm menor propensão a manifestar os sintomas do climatério em seus respectivos domínios do MRS: psicológico $(\mathrm{p}<0,01)$, somáticovegetativo $(p<0,01)$ e urogenital $(p<0,01)$. Este fator 
Tabela 1. Aspectos sociodemográficos das mulheres de meia idade atendidas nas Unidades Básicas em Natal, Rio Grande do Norte, 2011

\begin{tabular}{|c|c|c|c|c|c|c|c|}
\hline \multirow{3}{*}{ Variáveis } & \multicolumn{6}{|c|}{ Níveis de atividade física } & \multirow{3}{*}{ Valor $\mathrm{p}^{*}$} \\
\hline & \multicolumn{2}{|c|}{ Ativo } & \multicolumn{2}{|c|}{ Moderadamente ativo } & \multicolumn{2}{|c|}{ Sedentário } & \\
\hline & n & $\%$ & $n$ & $\%$ & n & $\%$ & \\
\hline Menor de 45 anos & 44 & 39,3 & 39 & 34,8 & 29 & 25,9 & 0,7 \\
\hline De 45 a 54 anos & 54 & 35,3 & 50 & 32,7 & 49 & 32,0 & \\
\hline Igual ou superior a 55 anos & 34 & 32,4 & 40 & 38,1 & 31 & 29,5 & \\
\hline Branca & 102 & 37,9 & 96 & 35,7 & 71 & 26,4 & 0,1 \\
\hline Não branca & 30 & 29,7 & 33 & 32,7 & 38 & 37,6 & \\
\hline \multicolumn{8}{|l|}{ Estado civil } \\
\hline Casado & 87 & 38,2 & 79 & 34,6 & 62 & 27,2 & 0,3 \\
\hline Divorciado & 10 & 33,3 & 9 & 30,0 & 11 & 36,7 & \\
\hline Não & 127 & 36,7 & 118 & 34,1 & 101 & 29,2 & 0,2 \\
\hline Sim & 5 & 20,8 & 11 & 45,8 & 8 & 33,3 & \\
\hline \multicolumn{8}{|l|}{ Nível educacional } \\
\hline Fundamental & 28 & 25,7 & 41 & 37,6 & 40 & 36,7 & 0,2 \\
\hline Médio & 67 & 37,9 & 60 & 33,9 & 50 & 28,2 & \\
\hline Superior & 37 & 44,0 & 28 & 33,3 & 19 & 22,6 & \\
\hline \multicolumn{8}{|l|}{ Renda mensal (IBGE) } \\
\hline Até 1 SM** & 14 & 31,8 & 16 & 36,4 & 14 & 31,8 & 0,01 \\
\hline 1 a 2 SM & 69 & 31,7 & 74 & 33,9 & 75 & 34,4 & \\
\hline
\end{tabular}

*valor $p$ associado ao teste do $\chi^{2}$ de Pearson; * ${ }^{*}$ SM: salário mínimo de referência no Censo Demográfico 2010 - IBGE.

Tabela 2. Teste de comparação múltipla de Tukey para as médias dos domínios do MRS segundo a classificação do nível de atividade física (IPAQ) das mulheres de meia idade, estudadas em Natal, Rio Grande do Norte, 2011

\begin{tabular}{|c|c|c|c|c|}
\hline Características & IPAQ & Média $^{1}$ & DP & Valor $p^{2}$ \\
\hline \multicolumn{5}{|l|}{ Qualidade de vida } \\
\hline & Ativa & $62,2^{\circ}$ & 8,9 & \\
\hline \multirow[t]{2}{*}{ WHOQOL-Bref } & Moderadamente ativa & $60,8^{\circ}$ & 9,5 & $<0,01$ \\
\hline & Sedentária & $56,7^{b}$ & 10,2 & \\
\hline \multicolumn{5}{|l|}{ Domínios do MRS } \\
\hline & Ativa & $4,6^{0}$ & 3,9 & \\
\hline \multirow{2}{*}{ Psicológico } & Sedentária & $7,5^{c}$ & 4,6 & \\
\hline & Ativa & $3,6^{0}$ & 3,1 & \\
\hline \multirow[t]{3}{*}{ Somático-vegetativo } & Moderadamente ativa & $5,0^{b}$ & 3,8 & $<0,01$ \\
\hline & Sedentária & $6,5^{c}$ & 3,9 & \\
\hline & Ativa & $2,8^{a}$ & 2,6 & \\
\hline
\end{tabular}

${ }^{1}$ Médias com letras iguais não são estatisticamente diferentes ao nível de $5 \% ;{ }^{2}$ Teste de comparação múltipla de Tukey; DP: desvio padrão.

pode explicar a melhor QV retratada por estas mulheres. Os resultados mostram a tendência de escores médios menores de sintomatologias à medida que aumenta o nível de atividade física realizado pelas mulheres. A prática de atividade física pode ser um determinante da QV das mulheres observadas.
O escore médio do WHOQOL-Bref ${ }^{17}$ para avaliar QV foi de 60,2, entretanto diferenças significantes foram observadas entre os três grupos do IPAQ ${ }^{16}(\mathrm{p}<0,01)$. Os escores médios de QV para as mulheres sedentárias, moderadamente ativas e muito ativas foram, respectivamente, 56,7; 60,8 e 62,2 (Tabela 2), diferindo significativamente 
Tabela 3. Análise comparativa do efeito da prática de atividade física na qualidade de vida das mulheres estudadas, Natal, Rio Grande do Norte, 2011

\begin{tabular}{|c|c|c|c|c|c|c|c|c|c|}
\hline \multirow{3}{*}{ Variável } & \multicolumn{8}{|c|}{ Qualidade de vida } & \multirow{3}{*}{ Valor $\mathrm{p}^{*}$} \\
\hline & \multicolumn{2}{|c|}{ Alta } & \multicolumn{2}{|c|}{ Média } & \multicolumn{2}{|c|}{ Baixa } & \multicolumn{2}{|c|}{ Total } & \\
\hline & n & $\%$ & $n$ & $\%$ & $n$ & $\%$ & $n$ & (\%) & \\
\hline IPAQ & & & & & & & & & \\
\hline Ativo & 54 & 40,9 & 46 & 34,9 & 32 & 24,3 & 132 & 35,7 & $<0,01$ \\
\hline Moderadamenteativo & 42 & 32,6 & 54 & 41,9 & 33 & 25,6 & 129 & 34,9 & \\
\hline Sedentário & 23 & 21,1 & 42 & 38,5 & 44 & 40,4 & 109 & 29,5 & \\
\hline
\end{tabular}

*Teste de Mantel-Haesnzel para dados ordinais.

as sedentárias daquelas que praticam alguma atividade, moderada ou vigorosamente ativas.

Avaliando a QV das mulheres com relação ao nível de atividade física que realizam (Tabela 3), os resultados apontaram para uma tendência das mais sedentárias à classificação em baixa QV, mostrando a forte associação entre a QV e o nível de atividade física $(\mathrm{p}<0,01)$ praticado pelas mulheres. Enquanto que naquelas mais ativas concentra 40,9\% classificadas em alta QV, nas sedentárias este percentual cai para $21,1 \%$, correspondendo a uma chance de melhor QV 2,6 vezes maior para as mulheres mais ativas.

\section{Discussão}

A atividade física tem apresentado impacto positivo na $\mathrm{QV}$, independente da idade, do sexo e do estado de saúde e tipo de atividade física ${ }^{20,21}$. No presente estudo, observou-se que a prática de atividade física teve um impacto positivo na saúde mental e física de mulheres de meia idade, atuando nos principais domínios da vida. Foi utilizado o WHOQOL-Bref ${ }^{17}$ para avaliar a QV, porque este instrumento, além de ser prático e de fácil utilização, já foi validado para português em mulheres brasileiras e principalmente porque foi considerado bem adequado para avaliar a população estudada ${ }^{17}$. Utilizando este instrumento juntamente com o IPAQ ${ }^{16}$ foram constatadas diferenças significativas na $\mathrm{QV}$ das mulheres pouco ativas, quando comparadas às moderadas e muito ativas $(\mathrm{p}<0,01)$, como pode ser observado na Tabela 3.

Os menores índices de QV foram observados nas mulheres sedentárias e os maiores nas muito ativas. Utilizando o mesmo instrumento (IPAQ), realizou-se um importante estudo brasileiro com 1.204 sujeitos (645 mulheres e 559 homens) com idade maior ou igual a 60 anos, no qual observou-se que os idosos de ambos os sexos, que praticavam atividade física, apresentavam significantemente melhores índices de habilidade sensorial e autonomia, além de apresentar significativamente maior QV global, independentemente da idade, do estado civil, da escolaridade e do nível socioeconômico. Neste estudo foi observado, especificamente nas mulheres, que os escores obtidos para o domínio da participação social foram significativamente maiores nas mulheres ativas e muito ativas, quando comparadas às sedentárias de forma semelhante ao que foi observado no presente estudo ${ }^{21}$.

Alguns estudos também têm fornecido suporte a esta associação, realçando a importância da prática de atividade física para mulheres mais velhas e de meia idade, melhorando inclusive a autoestima e o conceito que elas têm de si mesmas ${ }^{22,23}$. Neste estudo também com mulheres de meia idade, observou-se, de forma evidente $(\mathrm{p}<0,01)$, que houve maior prevalência da QV no grupo de mulheres mais ativas $(40,9 \%)$, quando comparadas ao grupo das sedentárias $(21,1 \%)$, correspondendo a uma chance 2,6 vezes maior de melhor QV entre as mulheres mais ativas (Tabela 3 ).

Em mulheres menopausadas, atividade física esteve associada à saúde psicossocial. Estudo recente realizado com 497 mulheres comparando as mulheres menos ativas com as mais ativas observou que a prática de atividade física esteve relacionada com a melhora dos sintomas de ansiedade e depressão, sintomas que provavelmente também tornam as mulheres menos dispostas a realizar exercícios $^{24}$. Acredita-se que as endorfinas liberadas pela mulher mais ativa durante a prática de atividade física possam por si só justificar o bem-estar e a visão mais positiva da vida destas mulheres ${ }^{25}$.

Diversas intervenções físicas e cognitivas têm sido realizadas para melhorar a cognição dos adultos de meia idade com resultados promissores ${ }^{26}$. A prática de atividade física tem demonstrado inúmeros benefícios físicos e mentais, incluindo também a melhora da cognição. Estudo recente que propôs avaliar o processo de atenção durante o envelhecimento constatou que a prática de tai chi chuan e dança contemporânea poderiam aumentar a flexibilidade cognitiva e atenção em adultos mais velhos, desta forma auxiliando-os na sua vida diária. Os autores sugerem ainda que a prática destas modalidades de atividade física poderia ser generalizada para condições patológicas, tais como a demência (Alzheimer), com a intenção de melhorar a capacidade cognitiva que naturalmente declina com a idade ${ }^{25}$. Estudo recente propõe ainda que a mudança no estilo de vida com incorporação de atividade física e dieta adequada poderia melhorar a saúde mental ${ }^{27}$. 
Por outro lado, deve-se considerar que a relação existente entre a prática de atividade física e melhora da qualidade em mulheres no período menopausal é complexa e pode envolver outros mecanismos, tais como: alterações neuroendócrinas, composição corporal, termorregulação e humor ${ }^{28,29}$, além de fatores biopsicossociais que não podem ser esquecidos e devem ser considerados na realização de futuros estudos sobre este tema ${ }^{8}$.
O presente estudo apresentou resultados interessantes, entretanto reconhece-se que existem limitações no mesmo. Os níveis de atividade física e os indícios de QV foram mensurados a partir de relatos da própria paciente, sem terem sido considerados outros parâmetros físicos, tais como a composição corporal e aptidão física. O modelo testado também não considerou outros aspectos biossociais. Portanto, deve-se ter cautela na generalização dos resultados.
1. Tumminello $M$, Miccichè $S$, Dominguez $L$, Lamura $G$, Melchiorre $M G$, Barbagallo $M$, et al. Happy aged people are all alike, while every unhappy aged person is unhappy in its own way. PloS One. $2011 ; 6(9): \mathrm{e} 23377$

2. Souza Santos CA, Dantas EE, Moreira MH. Correlation of physical aptitude; functional capacity, corporal balance and quality of life (QoL) among elderly women submitted to a postmenopausal physical activities program. Arch Gerontol Geriatr. $2011 ; 53(3): 344-9$.

3. Roush K. Prevention and treatment of osteoporosis in postmenopausal women: a review. Am J Nurs. 2011;111(8):26-35.

4. Meadows JL, Vaughan DE. Endothelial biology in the post-menopausal obese woman. Maturitas. $2011 ; 69(2): 120-5$.

5. Yanes LL, Reckelhoff JF. Postmenopausal hypertension. Am J Hypertens. $2011 ; 24(7): 740-9$.

6. Elavsky S, McAuley E. Physical activity and mental health outcomes during menopause: a randomized controlled trial. Ann Behav Med. 2007;33(2): 132-42.

7. Bize R, Johnson JA, Plotnikoff RC. Physical activity level and healthrelated quality of life in the general adult population: a systematic review. Prev Med. 2007;45(6):401-15.

8. Vallance JK, Murray TC, Johnson ST, Elavsky S. Quality of life and psychosocial health in postmenopausal women achieving public health guidelines for physical activity. Menopause. 2010;17(1): 64-71.

9. Wipfli BM, Rethorst CD, Landers DM. The anxiolytic effects of exercise: a meta-analysis of randomized trials and dose-response analysis. J Sport Exerc Psychol. 2008;30(4):392-410.

10. Tierney MC, Moineddin R, Morra A, Manson J, Blake JJ. Intensity of recreational physical activity throughout life and later life cognitive functioning in women. J Alzheimers Dis. 2010;22(4):1331-8.

11. Carvalho ED, Valadares ALR, Costa-Paiva LH, Pedro AO, Morais SS, Pinto-Neto AM. Atividade física e qualidade de vida em mulheres com 60 anos ou mais: fatores associados. Rev Bras Ginecol Obstet. 2010;32(9):433-40.

12. Asbury EA, Chandrruangphen $P$, Collins $P$. The importance of continued exercise participation in quality of life and psychological well-being in previously inactive postmenopausal women: a pilot study. Menopause. 2006;13(4):561-7.

13. WHO Scientific Group. Research on the menopause in the 1990s. World Health Organ Tech Rep Ser. 1996;866:1-107.

14. Instituto Brasileiro de Geografia e Estatística. Síntese de Indicadores Sociais: uma análise das condições de vida da população brasileira. Rio de Janeiro: IBGE; 2010. (Informação Demográfica e Socioeconômica, 27).
15. World Health Organization. Global recommendations on physical activity for health. Geneva: WHO; 2010.

16. Matsudo SM, Araújo T, Marsudo V, Andrade D, Andrade E, Oliveira LC, et al. Questionário Internacional de Atividade Física (IPAQ): estudo de validade e reprodutibilidade no Brasil. Rev Bras Ativ Fis Saúde. $2001 ; 6(2): 5-18$.

17. Fleck MPA, Louzada S, Xavier M, Chachamovich E, Vieira G, Santos $L$, et al. Aplicação da versão em português do instrumento abreviado de avaliação da qualidade de vida "WHOQOL-bref". Rev Saúde Pública. 2000;34(2):178-83.

18. Heinemann LA, Potthoff P, Schneider HP. International versions of the Menopause Rating Scale (MRS). Health Qual Life Outcomes. $2003 ; 1: 28$.

19. Ryan TA Jr, Joiner BF, Ryan BF. Minitab Reference Manual. Pennsylvania: Pennsylvania State University; 1981.

20. Potter R, Ellard D, Rees K, Thorogood M. A systematic review of the effects of physical activity on physical functioning, quality of life and depression in older people with dementia. Int J Geriatr Psychiatry. $2011 ; 26(10): 1000-11$

21. Guedes DP, Hatmann AC, Martini FA, Borges MB, Bernardelli R Jr. Quality of life and physical activity in a sample of Brazilian older adults. J Aging Health. 201 1. [ahead of print]

22. McAuley E, Konopack JF, Motl RW, Morris KS, Doerksen SE, Rosengren KR. Physical activity and quality of life in older adults: influence of health status and self-efficacy. Ann Behav Med. 2006;31(1):99-103.

23. Elavsky S. Physical activity, menopause, and quality of life: the role of affect and self-worth across time. Menopause. 2009; 16(2): 265-71.

24. Sternfeld B, Marcus R. Exercise. In: Lobo R, Kelsey J, Marcus $\mathrm{R}$, editors. Menopause: biology and pathobiology. San Diego: Academic Press; 2000. p. 495-504.

25. Spencer RL, Hruby VJ, Burks TF. Alteration of thermoregulatory set point with opioid agonists. J Pharmacol Exp Ther. 1990;252(2):696-705.

26. Coubard OA, Duretz $S$, Lefebvre V, Lapalus $P$, Ferrufino L. Practice of contemporary dance improves cognitive flexibility in aging. Front Aging Neurosci. $2011 ; 3: 13$.

27. Pronk NP, Katz AS, Gallagher J, Austin E, Mullen D, Lowry M, et al. Adherence to optimal lifestyle behaviors is related to emotional health indicators among employees. Popul Health Manag. $2011 ; 14:(2): 59-67$.

28. Daley A, Stokes-Lampard H, Macarthur C. Exercise for vasomotor menopausal symptoms. Cochrane Database Syst Rev. $2011 ; 11 ;(5): C D 006108$.

29. Chaouloff F. Physical exercise and brain monoamines: a review. Acta Physiol Scand. 1989;137(1):1-13 